\title{
Fluctuation in and Correlation Between Carotid Blood Flow Velocity and Systemic Hemodynamics During Pulmonary Surgery
}

\author{
Eriko Amano ${ }^{1}$, Kosuke Hamabe ${ }^{1}$, Takeshi Tateda ${ }^{2}$, and Soichiro Inoue ${ }^{1}$
}

(Received for Publication: August 10, 2018)

\begin{abstract}
Background: Carotid ultrasonography can be used as a minimally invasive method of evaluating systemic hemodynamics. We investigated carotid artery flow velocity during one-lung ventilation (OLV) to test our hypothesis that the measured values from carotid ultrasonography would positively correlate with global hemodynamic values, blood pressure, and arterial pressure-based cardiac output (APCO).

Methods: The study group comprised 43 consecutive patients who underwent pulmonary surgery between April and September 2017. Common carotid artery measurements on the same side as the non-dependent lung were obtained at 8 time points: before induction of general anesthesia, after tracheal intubation, after positional change, at 15, 30, 60, and 90 minutes after initiation of OLV, and 15 minutes after OLV. We measured peak systolic velocity (PSV) of the common carotid artery. Non-invasive systolic blood pressure (NISBP), non-invasive mean blood pressure (NIMBP), non-invasive diastolic blood pressure (NIDBP), systolic arterial pressure (SAP), mean arterial pressure (MAP), and diastolic arterial pressure (DAP), and APCO were recorded at the same points.

Results: PSV increased significantly after a change to the lateral decubitus position. Multiple regression analysis showed PSV was affected by APCO, SAP, age, and history of hypertension $\left(\mathrm{R}^{2}=0.23\right)$. APCO was the most affective factor $(\beta=8.35, \mathrm{SE}=1.60, \mathrm{t}=5.22, \mathrm{P}<0.0001)$. The second was $\mathrm{SAP}(\beta=-0.37, \mathrm{SE}=$ $0.08, \mathrm{t}=-4.75, \mathrm{P}<0.0001)$.
\end{abstract}

Conclusions: Carotid ultrasonography may be useful for evaluating systemic hemodynamics during pulmonary surgery.

\section{Key Words}

Carotid artery blood flow velocity, ultrasound, pulmonary surgery, one-lung ventilation

\section{Introduction}

Hemodynamic monitoring during the perioperative period is of importance because it allows to assess a patient's circulatory status including oxygen delivery and to detect physiological changes related to the surgery. Blood pressure measurement with a pneumatic cuff or an intra-arterial blood pressure measurement system is the standard of care for all patients under anesthesia, and for relatively unstable or critically ill patients, cardiac output (CO) is also measured. Maintenance of $\mathrm{CO}$ and maintenance of organ blood flow are essential for global oxygenation and tissue/organ oxygenation, respectively. CO measurement with pulmonary artery catheter based on Fick's law is considered accurate and has been the gold standard for CO measurement for several decades. However, it is an invasive procedure that can result in such accidental outcomes as arterial puncture, pulmonary artery rupture, hemothorax, pneumothorax, and local and systemic infections ${ }^{1-6}$.

Recent technical advances have provided for less invasive means of blood flow measurement. The use of the peripheral arterial waveform to calculate

1 Department of Anesthesiology, St. Marianna University School of Medicine

2 Department of Anesthesiology, Kawasaki Municipal Tama Hospital 
stroke volume (SV) has a history of over a century ${ }^{7}$. However, the early measurement devices require external calibration. The FloTrac/Vigileo ${ }^{\circledR}$ system (Edwards LifeSciences, Irvine, CA, USA), introduced in 2005, does not require external calibration and uses the arterial pressure signal obtained from a peripheral intra-arterial catheter as a standard to calculate SV and thereby $\mathrm{CO}$. Although these technologies provide for less invasive measurement of global hemodynamic values and $\mathrm{CO}$, they cannot be used to evaluate local hemodynamics or organ/tissue blood flow ${ }^{7}$.

Ultrasonography is widely used clinically for diagnosis and now commonly used for hemodynamic measurement. Physicians routinely use echocardiography preoperatively and/or intraoperatively. The primary advantage of echocardiography is that it is less invasive than modalities requiring vascular puncture. Carotid ultrasonography has also come into common use as a means of clinical evaluation. Carotid atherosclerosis, for example, is evaluated on the basis of carotid intima-media thickness, plaque quality, or carotid flow velocity ${ }^{8,99}$. Results of several studies have pointed toward the use of carotid ultrasonography as a hemodynamic measurement tool. Ma et al. reported correlation between carotid blood flow and $\mathrm{CO}$ in patients undergoing right heart catheterization $^{10)}$. Marik et al. reported significantly positive correlation between percent change in carotid blood flow and percent change in the stroke volume index $(\mathrm{SVI})^{11)}$. According to Song et al., carotid artery peak velocity variation correlates with pulse pressure variation $(\mathrm{PPV})^{12)}$. They concluded that carotid ultrasonography may be useful for non-invasive hemodynamic evaluation tool. Hence, we think that these findings suggest carotid ultrasonography is a candidate for intraoperative circulatory monitoring. To the best of our knowledge, however, there are no reports of use of carotid Doppler ultrasonography for hemodynamic monitoring in patients undergoing surgery under general anesthesia and one-lung ventilation (OLV).

We hypothesized that the measured values from carotid ultrasonography correlate positively with global hemodynamic values, blood pressure, and $\mathrm{CO}$, and that a patient's age and arterial stiffness may influence the carotid artery flow velocity. The aim of the study described herein was to test this hypothesis during pulmonary surgery requiring OLV.

\section{Patients and Methods}

Entered into the study were 48 consecutive pa- tients undergoing pulmonary surgery for lung cancer at St. Marianna University School of Medicine Hospital between April and September 2017. Exclusion criteria were a diagnosis of cerebral infarction, carotid artery stenosis, chronic or paroxysmal atrial fibrillation, aortic regurgitation, or interstitial pneumonia. The study was approved by the institutional review board of St. Marianna University School of Medicine (approval number: 3485), and written informed consent was obtained from all patients involved.

\section{Anesthesia induction, arterial cannulation, and ventilation}

All surgeries were performed under combined epidural-general anesthesia, and none of the patients was pre-medicated. Standard electrocardiographic monitoring, arterial oxygen saturation monitoring, and non-invasive blood pressure monitoring were performed. Before the induction of general anesthesia, an epidural catheter was placed in the $T 7 / 8$ or T8/9 intervertebral space, and the patient was kept supine for 5 minutes. The general anesthesia was then induced by intravenous administration of remifentanil at $0.35-0.5 \mu \mathrm{g} \mathrm{kg}^{-1} \mathrm{~h}^{-1}$, followed by propofol $1-2 \mathrm{mg} \mathrm{kg}^{-1}$ and rocuronium $0.8-1 \mathrm{mg} \mathrm{kg}^{-1}$. A leftsided double-lumen endotracheal tube (BronchoCath $^{\circledR}$, Covidien, Mansfield, MA, USA) was inserted. The radial artery on the same side as the dependent lung was cannulated and connected via FloTrac ${ }^{\circledR}$ sensor to an arterial contour analysis system (EV $1000^{\circledR}$, Edwards Lifesciences, Irvine, CA, USA) for continuous measurement of arterial pressure and arterial pressure-based cardiac output (APCO). APCO is computed as follows: pulse rate $\times$ APsd $\times \mathrm{K}$. APsd is the standard deviation of arterial pressure and $\mathrm{K}$ is an autocalibration factor from a proprietary multivariate equation, which mainly contains arterial compliance and peripheral vascular resistance (PVR) ${ }^{13}$. Anesthesia was maintained with $4-5 \%$ desflurane or $4-6 \mathrm{mg}$ $\mathrm{kg}^{-1} \mathrm{~h}^{-1}$ propofol. The fraction of inspiratory oxygen $\left(\mathrm{FIO}_{2}\right)$ during OLV was 1.0. At the start of two-lung ventilation, the $\mathrm{FIO}_{2}$ was 0.6. Pressure-control mechanical ventilation was set to maintain a tidal volume of 6-7 mL per ideal body weight $\left(\mathrm{kg}^{-1}\right)$ and positive end-expiratory pressure of $4 \mathrm{~cm} \mathrm{H}_{2} \mathrm{O}$. The respiratory rate was set to $8-10$ per minute to maintain normocapnia.

\section{Hemodynamic measurements}

Hemodynamic measurements were obtained at the following time points: 
1. Before the induction of general anesthesia, i.e., 5 minutes after insertion of the epidural catheter. This was considered the baseline measurement.

2. Just after insertion of the bronchial tube and the radial artery cannula.

3. Just after the patient is moved from the supine to the lateral decubitus position.

4. Fifteen, 30, 60, and 90 minutes after the initiation of OLV.

5. Fifteen minutes after the conclusion of OLV.

These time points were chosen so that the effects of anesthesia, positional change, and duration of OLV on carotid blood flow velocity and systemic hemodynamics could be evaluated. All common carotid artery measurements were obtained by the same examiner with a GE Vivid i ${ }^{\circledR}$ Doppler ultrasound scanner and a linear probe (12L, 5.0-13.0 MHz: GE Healthcare, Tokyo, Japan). At the time of the first measurement, the short-axis view was obtained. The long-axis view was subsequently obtained and checked for the presence of severe stenosis. Severe stenosis was defined as peak systolic velocity (PSV) $>200 \mathrm{~cm} /$ second (indicative of $\geq 70 \%$ stenosis according the North American Symptomatic Endarterectomy Trial (NASCET) criteria) ${ }^{14}$. Doppler measurement was performed about $2 \mathrm{~cm}$ proximal to the carotid bulb. The angle cursor was placed parallel to the vessel, and the beams were adjusted to an angle of $<60^{\circ}$. PSV of the common carotid artery on the same side as the non-dependent lung was measured at each time point listed above. PSV, i.e., maximum blood flow velocity in the systolic phase, is a commonly used parameter of carotid blood flow velocity, and its variation is sometimes calculated ${ }^{12), 20-22)}$. Because hemodynamic monitoring during surgery requires not only accuracy but also swiftness, we regarded the measured value is important and did not convert the measured value into the rate of variability or blood flow. Systemic hemodynamic variables, i.e., APCO, non-invasive systolic blood pressure (NISBP), non-invasive mean blood pressure (NIMBP), non-invasive diastolic blood pressure (NIDBP), systolic arterial pressure (SAP), mean arterial pressure (MAP), and diastolic arterial pressure (DAP), were also recorded at each time point, and all measurements were obtained in the absence of atrial fibrillation and other arrhythmias.

\section{Statistical analyses}

Continuous variables are shown as median and interquartile range or mean \pm standard deviation.
Changes in carotid and systemic hemodynamic variables were analyzed by repeated measures analysis of variance and Tukey's post hoc test. Relations between common carotid artery flow velocity and the systemic hemodynamic variables were analyzed by means of stepwise multiple linear regression. Variables were selected by the Akaike information criterion method. The final variables included were SAP, APCO, age, and hypertension. JMP ${ }^{\circledast}$ Pro version 13.0.0 (SAS Institute Inc, Cary, NC, USA) was used for all statistical analyses, and $\mathrm{P}<0.05$ was considered statistically significant.

\section{Results}

The final study group comprised 43 patients who underwent pulmonary surgery. Five patients were dropped from the study because of displacement of endobronchial tube. The tube was adjusted, but this affected the OLV time. Characteristics of the 43 patients are shown in Table 1.

\section{Change in hemodynamic variables in the 43 pa- tients}

PSV increased significantly above the baseline values just after lateral decubitus positioning $(\mathrm{P}<$ 0.0001). APCO increased steadily during OLV, and NISBP, NIMBP, and NIDBP decreased significantly below the baseline values after induction of general anesthesia (Table 2).

Table 3 shows the results of multiple linear regression analysis used to determine the relation between PSV and systemic hemodynamic covariates, with adjustment for age and hypertension. Positive correlation was found between PSV and APCO, and negative correlation was found between PSV and SAP (coefficient of determination $\left(\mathrm{R}^{2}\right)=0.232[\mathrm{P}<$ 0.0001]).

\section{Discussion}

In this study, we investigated the relation between ultrasonically determined carotid blood flow velocity and systemic hemodynamics. We found significant correlation between PSV and APCO. The regression coefficient for the relation between PSV and APCO was 8.35, and that for the relation between PSV and SAP was -0.37 , showing that both APCO and SAP are the factors determining PSV. The increase in cardiac contractility or decrease in PVR augments $\mathrm{CO}$ and thus enhances the velocity of systemic blood flow. Hence, the increase in PSV or APCO indicates augmentation of cardiac contractil- 
Table 1. Patient Characteristics $(n=43)$

\begin{tabular}{lc}
\hline Male/female & $25 / 18$ \\
Age (years) & $65 \pm 9$ \\
Height (m) & $1.63 \pm 0.09$ \\
Weight (kg) & $59 \pm 10$ \\
BMI (kg/m ${ }^{2}$ ) & $22.2 \pm 2.8$ \\
ASA PS (1/2) & $3 / 40$ \\
Surgical side (left/right) & $16 / 27$ \\
Resection site (upper/middle/lower lobe) & $29 / 3 / 11$ \\
Duration of surgery (minutes) & $147 \pm 48$ \\
Duration of anesthesia (minutes) & $228 \pm 50$ \\
Duration of OLV (minutes) & $140 \pm 48$ \\
Maintenance of anesthesia (desflurane/propofol) & $23 / 20$ \\
Hypertension (\%) & 32 \\
\hline Mean (SD) value or number of patients is shown unless otherwise indicated. \\
BMl, body mass index; OLV, one-lung ventilation; ASA PS, American \\
Society of Anesthesiologists physical status. \\
Hypertension; Patients with a history of hypertension.
\end{tabular}

Table 2. Common Carotid Artery Blood Flow Velocity and Systemic Hemodynamic Variables

\begin{tabular}{|c|c|c|c|c|c|c|c|c|}
\hline & Before anesthesia & After anesthesia & Lateral positioning & $15 \mathrm{~min}$ of $\mathrm{OLV}$ & $30 \mathrm{~min}$ of OLV & $60 \mathrm{~min}$ of OLV & $90 \mathrm{~min}$ of OLV & 15 min after OLV \\
\hline $\mathrm{PSV}(\mathrm{cm} / \mathrm{s})$ & $61.4(53.8-72.4)$ & $69.1(61.4-84.5)$ & $82.3(68.6-92.2)^{*}$ & $72.8(60.9-96.5)^{*}$ & $83.4(71.3-104.2)^{*}+\dagger$ & $97.8(76.0-108.6)^{*,+.+. \S}$ & $84.5(71.9-99.8)^{*}+\dagger$ & $88.9(68.0-99.8)^{*}+\dagger$ \\
\hline APCO (L/min) & N.A. & $3.5(3.3-3.7)$ & $3.5(3.1-3.8)$ & $3.7(3.2-4.1)$ & $4.1(3.5-4.4)^{\ddagger . \S}$ & $4.3(3.8-4.6)^{\dagger . \neq . \S}$ & $4.1(3.8-4.5)^{\ddagger . \S}$ & $3.9(3.5-4.6)^{\ddagger . \S}$ \\
\hline $\mathrm{NISBP}(\mathrm{mmHg})$ & $139(125-152)$ & $86(76-110)^{*}$ & $96(80-105)^{*}$ & $104(87-121)^{*}$ & $100(81-112)^{\circ}$ & $94(82-106)^{*}$ & $91(81-102)^{*}$ & $88(76-102)^{*}, \S$ \\
\hline $\operatorname{NIMBP}(\mathrm{mmHg})$ & $99(90-105)$ & $66(56-78)^{\circ}$ & $69(58-80)^{*}$ & $74(57-87)^{*}$ & $70(58-83)^{\circ}$ & $70(56-78)^{*}$ & $64(57-78)^{\circ}$ & $67(54-78)^{* .8}$ \\
\hline NIDBP $(\mathrm{mmHg})$ & $80(66-87)$ & $56(47-64)^{\circ}$ & $53(45-67)^{\circ}$ & $60(46-71)^{\circ}$ & $56(46-66)^{\circ}$ & $55(43-64)^{\circ}$ & $53(44-67)^{\circ}$ & $53(40-67)^{\circ}$ \\
\hline $\mathrm{SAP}(\mathrm{mmHg})$ & N.A. & $100(82-125)$ & $95(88-107)$ & $110(93-122)^{\ddagger}$ & $100(88-115)$ & $100(90-111)$ & $99(93-105)$ & $92(86-108)^{\S}$ \\
\hline $\operatorname{MAP}(\mathrm{mmHg})$ & N.A. & $66(54-78)$ & $68(57-76)$ & $76(64-90)^{f}$ & $70(61-82)$ & $68(62-77)$ & $70(64-75)$ & $67(60-74)^{\S}$ \\
\hline $\mathrm{DAP}(\mathrm{mmHg})$ & N.A. & $50(42-59)$ & $55(46-60)$ & $59(50-68)^{t . \neq}$ & $55(47-63)$ & $53(47-61)^{\S}$ & $54(49-59)$ & $53(48-58)^{\S}$ \\
\hline
\end{tabular}

Median (interquartile range) values are shown

OLV, one-lung ventilation; PSV, peak systolic velocity; APCO, arterial pressure-based cardiac output; NISBP, non-invasive systolic blood pressure; NIMBP, non-invasive mean blood pressure; NIDBP, noninvasive diastolic blood pressure; SAP, systolic arterial pressure; MAP, mean arterial pressure; DAP, diastolic arterial pressure; N.A., not available.

$\mathrm{P}<0.05$ *vs before induction of anesthesia, ${ }^{\mathrm{t} v s}$ after induction of anesthesia, ${ }^{7} \mathrm{vs}$ after lateral positioning, ${ }^{5} \mathrm{vs} 15$ minutes of OLV, "vs 30 minutes of OLV, "vs 60 minutes of OLV

"vs 90 minutes of OLV, " vs 15 minutes after OLV.

Table 3. Multiple regression analysis for PSV

\begin{tabular}{lccc}
\hline & $\begin{array}{c}\text { Adjusted Regression } \\
\text { Coefficient }\end{array}$ & SE & P value \\
\hline interception & 123.59 & 15.10 & $<.0001$ \\
APCO & 8.35 & 1.60 & $<.0001$ \\
SAP & -0.37 & 0.08 & $<.0001$ \\
age & -0.53 & 0.16 & 0.0012 \\
HT & 3.34 & 1.56 & 0.0335 \\
\hline
\end{tabular}

PSV, peak systolic velocity; SE, standard error; HT, hypertension;

APCO, arterial pressure-based cardiac output; SAP, systolic arterial pressure;

N.A., not available.

ity. Furthermore, we found inverse correlation between carotid blood flow velocity and SAP. A possible explanation for this finding is the relation between PVR and cardiac contractility. A decrease in PVR causes a reduction in cardiac afterload and augments CO, and this may lead to an increase in PSV.
Therefore, blood flow velocity is affected by cardiac contractility and also by arterial stiffness and expan$\operatorname{sion}^{15-17)}$.

In our multiple linear regression analysis, we adjusted for age and hypertension. Because a patient's age and arterial stiffness influence the velocity of ar- 
terial blood flow, and because atherosclerosis is not common in relatively young patients, we hypothesized that arterial blood flow velocity is a direct reflection of arterial blood flow volume in these patients. We found the regression coefficient between PSV and age to be $-0.53(\mathrm{P}=0.0012)$. That is, PSV decreases with age. This finding supports our hypothesis. Conversely, we found the regression coefficient between PSV and hypertension to be 3.34 ( $\mathrm{P}=$ $0.033)$. This finding may pertain to anesthesia. During general anesthesia, blood pressure decreases more in patients with hypertension that it does in patients without hypertension. So, a reduction in vascular resistance may increase cardiac contractility and PSV.

Our results point to measurement of carotid hemodynamic variables may be one of alternative means of measuring systemic hemodynamics. However, the coefficient of determination indicated a weak relation between PSV and systemic hemodynamic variables. A plausible explanation of this weak relation is that we measured not carotid flow volume but carotid flow velocity. Measuring carotid flow velocity and diameter simultaneously allowed us to estimate carotid artery flow volume. Both stiffness and the diameter of the carotid artery increase with age, so carotid flow velocity is decreased in elderly persons ${ }^{18}$. However, carotid flow volume per minute is constant and not influenced by age. Carotid blood flow volume is calculated as follows: $\pi \times$ (common carotid artery diameter $)^{2} / 4 \times$ velocity time integral $\times$ heart rate. If we had measured the diameter of the carotid artery, converted it to calculate the flow volume, and then examined correlation, we might have found a stronger correlation between carotid blood flow and systemic hemodynamic variables in our linear regression model.

The hemodynamic change we observed during pulmonary surgery may seem somewhat dramatic. However, we know that insertion of endobronchial tube causes hypertension, and positional change during anesthesia causes hypotension. There has not been a study of carotid blood flow velocity and systemic hemodynamics during pulmonary surgery, so, we evaluated fluctuations in carotid flow velocity and hemodynamics. The fluctuation in PSV paralleled fluctuations in systemic hemodynamics. This finding suggests that carotid flow velocity can be used to estimate $\mathrm{CO}$, and thus carotid ultrasonography may be useful for evaluating systemic hemodynamics.

The radial artery catheter was placed in our pa- tients after the induction of general anesthesia, so we did not analyze the relation between carotid flow velocity and APCO during induction of anesthesia. With the exception in the initial change from the baseline measurement, there was no significant change in PSV or systemic hemodynamics during anesthesia. In the interest of patient safety, changes in systemic hemodynamics were, as much as possible, kept to a minimum by the anesthesiologist.

One clear finding, however, was that carotid flow velocity and APCO did not change clinically during OLV, although $\mathrm{FIO}_{2}$ remained at 1.0.

Carotid flow velocity on the non-dependent lung side did not decrease when our patients were changed to the lateral decubitus position, though a head-position-based decrease in cervical blood flow velocity during thyroidectomy was reported ${ }^{19}$. After general anesthesia is induced with an agent such as propofol, PVR decreases, as do SAP. The decrease in PVR leads to a reduction in cardiac afterload, and thus cardiac contractility and $\mathrm{CO}$ increase. The increase in APCO in our study might result in an increase in carotid flow velocity.

Results of several studies have indicated that carotid ultrasonography is useful for evaluating carotid hemodynamics. Kleczyński et al. showed a significant drop in carotid velocity in patients with severe aortic stenosis when moving from a sitting position to standing position, and it may predict the risk of syncope $^{20)}$. There are many studies concerning fluids responsiveness and hemodynamic monitoring. Nowadays, fluid responsiveness is a standard of fluid management during the perioperative period. The measurement of fluid responsiveness is performed with several methods, such as SV variation or pulse pressure variation ${ }^{11), 12,21-23)}$. Carotid hemodynamic measurement applies to this fluid responsiveness. Marik et al. showed an increase in carotid blood flow after passive leg raising in responders and a strong correlation between change in SVI and change in carotid blood flow ${ }^{11}$. Furthermore, Buget et al. reported positional change from a supine to beach chair position was associated with a decrease in cerebral blood flow measured by Doppler ultrasonography and MAP. In this report, they measured the time averaged mean velocity of the internal carotid artery ${ }^{24)}$. If they had measured common carotid artery, carotid velocity may have decreased in the same manner. These studies showed that carotid hemodynamic measurement is a powerful and less invasive form of hemodynamic monitoring. 
Carotid artery blood flow velocity correlated with APCO. The velocity alone, without conversion to flow volume, might be useful for estimating APCO. Our findings suggest that carotid ultrasonography may be a candidate for monitoring patients' hemodynamics during pulmonary surgery. However, further study is needed before we can say with certainty that carotid ultrasonography can be used clinically to evaluate systemic hemodynamics during anesthesia.

Our study findings should be interpreted in light of the following limitations. First, we did not catheterize the radial artery before anesthesia because it is beyond our routine practice for patient monitoring, so our APCO and SAP data pertain only to the time period during which patients were under anesthesia. Further analysis of the relation between APCO and PSV would require measurement of the APCO when the patient is awake. Second, variables were measured only once per period by one examiner, and the reliability of those measurements was not analyzed. Third, the diameter of the carotid artery and flow velocity on the dependent lung side were not measured. Future investigation should include a comparison of these values between the non-dependent and dependent lung sides. Carotid ultrasonography on the dependent lung side is difficult to perform, but such measurements will provide us with further insight. A decrease in flow velocity on the dependent lung side might be compensated for by an increase on the nondependent lung side. Further investigation might help elucidate the mechanism underlying hypoxic vasoconstriction during OLV.

\section{Conclusion}

In conclusion, we found a positive correlation between carotid artery blood flow velocity measured by Doppler ultrasonography and APCO. This result suggests that carotid flow velocity can be used to estimate $\mathrm{CO}$, and thus carotid ultrasonography may be a candidate for evaluating systemic hemodynamics during pulmonary surgery.

\section{Acknowledgement}

We thank the ultrasonographers in the ultrasound examination center of St. Marianna University Hospital. We also thank Prof. Tina Tajima for her help in reviewing and editing the manuscript.

\section{References}

1) Bhandary R, Chakka U. Care and monitoring of the anaesthetized patient. Surgery 2016; 34: 79 83.

2) Cove ME, Pinsky MR. Perioperative hemodynamic monitoring. Best Prac Res Anaesthesiol 2012; 26: 453-462.

3) Teboul JL, Saugel B, Cecconi M, De Backer D, Hofer CK, Monnet X, Perel A, Pinsky MR, Reuter DA, Rhodes A, Squara P, Vincent JL, Scheeren TW. Less invasive hemodynamics monitoring in critically ill patients. Intensive Care Med 2016; 42: 1350-1359.

4) Gilbert M. Central venous pressure and pulmonary artery pressure monitoring. Anaesth Intensive Care 2018; 19: 189-193.

5) Wong AV, Arora N, Olusanya O, Sharif B, Lundin RM, Dhadda A, Clarke S, Siviter R, Argent M, Denton G, Dennis A, Day A, Szakmany T; First Intensive Care National Audit Project (ICNAP-1) group. Insertion rates and complications of central lines in the UK population: a pilot study. J Intensive Care Soc 2018; 19: 19-25.

6) McGee DC, Gould MK. Preventing complications of central venous catheterization. N Engl J Med 2003; 348: 1123-1133.

7) Slagt C, Malagon I, Groeneveld AB. Systematic review of uncalibrated arterial pressure waveform analysis to determine cardiac output and stroke volume variation. Br J Anaesth 2014; 112: 626-637.

8) Stein JH, Korcarz CE, Post WS. Use of carotid ultrasound to identify subclinical vascular disease and evaluate cardiovascular disease risk: summary and discussion of the American Society of Echocardiography consensus statement. Prev Cardiol 2009; 12: 34-38.

9) Reutern GM, Goertler MW, Bornstein NM, Sette MD, Evans DH, Hetzel A, Kaps M, Perren F, Razumovky A, Shiogai T, Titianova E, Traubner P, Venketasubramanian N, Wong LKS, Yasaka M. Grading carotid stenosis using ultrasonic methods. Stroke 2012; 43: 916-921.

10) Ma IWY, Caplin JD, Azad A, Wilson C, Fifer MA, Bagchi A, Liteplo AS, Noble VE. Correlation of carotid blood flow and corrected carotid flow time with invasive carotid output measurements. Crit Ultrasound J 2017; 9: 1-8.

11) Marik PE, Levitov A, Young A, Andrews L. The use of bioreactance and carotid Doppler to determine volume responsiveness and blood flow redistribution following passive leg raising in hemodynamically unstable patients. Chest 2013; 
143: 364-370.

12) Song Y, Kwak Y, Song JW, Kim YJ, Shim JK. Respirophasic carotid artery peak velocity variation as a predictor of fluid responsiveness in mechanically ventilated patients with coronary artery disease. Br J Anaesth 2014; 113: 61-66.

13) De Backer D, Marx G, Tan A, Junker C, Van Nuffelen M, Hüter L, Ching W, Michard F, Vincent JL. Arterial pressure-based cardiac output monitoring: a multicenter validation of the thirdgeneration software in septic patient. Intensive Care Med 2011; 37: 233-240.

14) Jahromi AS, Cinà CS, Liu Y, Clase CM. Sensitivity and specificity of color duplex ultrasound measurement in the estimation of internal carotid artery stenosis: A systematic review and meta-analysis. J Vasc Surg 2005; 41: 962-972.

15) Tomoto $T$, Maeda S, Sugawara J. Influence of blood flow velocity on arterial distensibility of carotid artery in healthy men. J Physiol Sci 2017; 67: 191-196.

16) Patel AK, Suri HS, Singh J, Kumar D, Shafique S, Nicolaides A, Jain SK, Saba L, Gupta A, Laird JR, Giannopoulos A, Suri JS. A review on atherosclerotic biology, wall stiffness, physics of elasticity, and its ultrasound-based measurement. Curr Atheroscler Rep 2016; 18: 1-12.

17) Heffernan KS, Lefferts WK, Augustine JA. Hemodynamic correlates of late systolic flow velocity augmentation in the carotid artery. Int $\mathbf{J}$ Hypertens 2013; 2013: 1-7.

18) Mannami T, Baba S, Ogata J. Potential of carotid enlargement as a useful indicator affected by high blood pressure in a large general population of a Japanese city. Stroke 2000; 31: 2958-2965.

19) Saraçoğlu A, Altun D, Yavru A, Aksakal N, Sormaz İC, Camc1 E. Effects of head position on cerebral oxygenation and blood flow velocity during thyroidectomy. Turk J Anaesthesiol Reanim 2016; 44: 241-246.

20) Kleczyński P, Petkow Dimitrow P, Dziewierz A, Surdacki A, Dudek D. Decreased carotid and vertebral arterial blood-flow velocity in response to orthostatic unload in patients with severe aortic stenosis. Cardiol J 2016; 23: 393-401.

21) Kim DH, Shin S, Kim N, Choi T, Choi SH, Choi YS. Carotid ultrasound measurements for assessing fluid responsiveness in spontaneously breathing patients: corrected flow time and respirophasic variation in blood flow peak velocity. Br J Anaesth 2018; 121: 541-549.

22) Hossein-Nejad H, Mohammadinejad P, LessanPezeshki M, Davarani SS, Banaie M. Carotid artery corrected flow time measurement via bedside ultrasonography in monitoring volume status. J Crit Care 2015; 30: 1199-1203.

23) Jalil B, Thompson $P$, Cavallazzi R, Marik P, Mann J, El-Kersh K, Guardiola J, Saad M. Comparing changes in carotid flow time and stroke volume induced by passive leg raising. Am J Med Sci 2018; 355: 168-173.

24) Buget MI, Atalar AC, Edipoglu IS, Sungur Z, Sivrikoz N, Karadeniz M, Saka E, Kucukay S, Senturk MN. Patient state index and cerebral blood flow changes during shoulder arthroscopy in beach chair position. [in Portuguese with English abstract] Rev Bras Anestesiol 2016; 66: 470-474. 